\title{
Consideraciones taxonómicas de Callistochiton pulchellus (Gray, 1828) (Mollusca, Placophora) en Iquique, norte de Chile
}

\author{
Moisés A. Aguilera \\ Estación Costera de Investigaciones Marinas, Las Cruces, Departamento de Ecología \\ Pontificia Universidad Católica de Chile, Casilla114-D Santiago, Chile
}

\begin{abstract}
RESUMEN. Se presenta información taxonómica de Callistochiton pulchellus (Gray, 1828) recolectado entre Iquique y el sector de Pabellón de Pica en la zona norte de Chile. Cada sitio donde se realizaron los muestreos, estuvo separado en al menos $1 \mathrm{~km}$. La especie se observó en tres de los trece sitios de muestreo (23\%) y sólo bajo bolones empotrados en la arena. Se determinó un nuevo límite sur para esta especie en el sector de Chanavayita, a 54 km al sur de Iquique. Se señalan los rasgos más importantes de su hábitat y las diferencias morfológicas más relevantes con Calloplax vivipara (Plate, 1902), que tiene una forma y estructuras del tegmentum similares a Callistochiton pulchellus. Estas diferencias se refieren principalmente al mayor número de los radios de la placa cefálica de C. pulchellus ( 12 radios) en comparación a los observados en $C$. vivipara ( 9 radios), y además a que las placas centrales de $C$. pulchellus son más rectangulares que en esta última.
\end{abstract}

Palabras clave: Callistochiton pulchellus, caracteres taxonómicos, distribución geográfica, Iquique, Chile.

\section{Taxonomic considerations on Callistochiton pulchellus (Gray, 1828) (Mollusca, Placophora) at Iquique, northern Chile}

\begin{abstract}
Information is presented on taxonomic traits of Callistochiton pulchellus (Gray, 1828) collected at

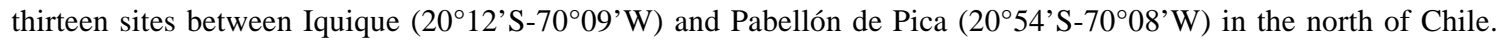
Sampling sites were at least one $\mathrm{km}$ apart. The species was found at only three of the thirteen sampling sites $(23 \%)$, and only underneath boulders sunken in sand. A new southern limit for this species was recorded in Chanavayita, 54 $\mathrm{km}$ south of Iquique. The most important characteristics of its habitat are presently discussed, as well as the the most relevant morphological differences of this species in comparison with Calloplax vivipara (Plate, 1902) which it closely resembles in form and tegmental ornamentation. C. pulchellus had a greater number of radial lines on the cephalic plate $(\$ 12)$ than $C$. vivipara $(4)$. It was also noted that the central plates in the former species were more rectangular than those of the latter species.
\end{abstract}

Key words: Callistochiton pulchellus, taxonomic traits, geographic distribution, Iquique, Chile.

Callistochiton pulchellus (Gray, 1828) es una de las 58 especies de chitones presentes en Chile (Kaas \& van Belle, 1994; Valdovinos, 1999). La distribución de esta especie se había señalado entre Guaymas, México $\left(27^{\circ} \mathrm{N}\right)$ y Arica, Chile $\left(20^{\circ} \mathrm{S}\right)$ (Keen, 1971), pero otros autores (Kaas \& van Belle, 1994) seña- lan su presencia sólo entre las localidades de Islay (Perú) y Pozo Toyo (Chile) e islas Galápagos. En Chile sólo se conocen reportes aislados de esta especie. Ferreira (1979) la menciona en la zona intermareal del sector de Pozo Toyo, a $23 \mathrm{~km}$ al sur de Iquique, pero en un estudio anterior realizado por 
Marincovich (1973) en esta misma localidad no menciona la presencia de esta especie. Así también, Leloup (1956) señala que esta especie no fue recolectada por la expedición sueca "Lund" (1948-1949) cuya colección de chitones chilenos fue abundante y diversa. Aunque también se ha mencionado su presencia en Arica, Chile (Boudet, 1945; Stuardo, 1959), al parecer esta información no fue respaldada por recolectas de la especie. Así, es evidente la falta de información acerca de $C$. pulchellus, la única especie representante de este género en la costa chilena.

En este trabajo, se entrega información sobre la presencia de $C$. pulchellus entre Iquique $\left(20^{\circ} 12^{\prime} \mathrm{S}\right.$ $\left.70^{\circ} 09^{\prime} \mathrm{W}\right)$ y el sector de Pabellón de Pica (2054'S$70^{\circ} 08^{\prime} \mathrm{W}$ ), norte de Chile. Además, se presentan los caracteres taxonómicos más relevantes para su identificación.

Entre mayo y julio de 2004 se realizó una serie de muestreos que consideraron tres sitios de la zona costera de Iquique y otros 10 sectores comprendidos entre ésta y Pabellón de Pica, $75 \mathrm{~km}$ al sur de Iquique. Los sitios de muestreo estuvieron separados por al menos $1 \mathrm{~km}$ (Tabla 1). En cada sector se realizó la búsqueda de la especie en zonas de bolones y plataformas rocosas (4 horas de muestreo aproximadamente, realizados mensualmente en cada sec- tor), considerando desde la zona intermareal alta hasta $1 \mathrm{~m}$ de profundidad. La identificación de los individuos se realizó siguiendo las características taxonómicas señaladas por Ferreira (1979) y Kaas \& van Belle (1994). En cada sitio donde se observó la especie se tomó una muestra de al menos 10 individuos de tallas entre 5 a $13 \mathrm{~mm}$ de longitud para un análisis taxonómico posterior. Los individuos recolectados se fijaron en etanol al $95 \%$.

C. pulchellus se registró con una frecuencia relativa de $23 \%$, es decir, en 3 de los 13 sitios considerados (calculado mediante la relación; número de localidades en que se observó la especie dividido por el total de localidades, y esto llevado a porcentaje). Los individuos sólo se observaron bajo bolones y cantos rodados empotrados en conchuela fina en zonas protegidas del oleaje (Tabla 1). Los bolones donde se observó $C$. pulchellus, presentaron un mosaico de especies sésiles constituido por briozoos, esponjas y poliquetos spirórbidos, el cual fue similar entre estos sitios de muestreo (datos aun no publicados por el autor). Al parecer estos organismos constituirían parte de la dieta de este chitón, dado que al analizar el contenido estomacal de $C$. pulchellus se observaron restos de estos organismos junto a una variada gama de diatomeas.

Por otra parte, se observó que C. pulchellus co-

Tabla 1. Listado de los sectores de muestreo de Callistochiton pulchellus (Gray, 1828) entre Iquique y Pabellón de Pica (75 km de rango espacial), y presencia/ausencia en zonas protegidas y expuestas al oleaje en dichos sectores. B: presencia en zona de bolones y cantos rodados. $\mathrm{X}$ : ausencia.

Table 1. List of Callistochiton pulchellus (Gray, 1828) sample sites between Iquique and Pabellón de Pica (75 km of spatial range), and presence/absence in protected and exposed zones in these sites. B: presence in boulders fields; X: absence.

\begin{tabular}{lccc}
\hline Sector & $\begin{array}{c}\text { Zona } \\
\text { protegida }\end{array}$ & $\begin{array}{c}\text { Zona } \\
\text { expuesta }\end{array}$ & $\begin{array}{c}\text { Distancia aproximada } \\
\text { de Iquique (km) }\end{array}$ \\
\hline Bellavista & $\mathrm{X}$ & $\mathrm{X}$ & 0 \\
Península de Cavancha & $\mathrm{X}$ & $\mathrm{X}$ & 0 \\
Primeras Piedras & $\mathrm{X}$ & $\mathrm{X}$ & 0 \\
Huayquique & $\mathrm{X}$ & $\mathrm{X}$ & 3,0 \\
Bajo Molle & $\mathrm{X}$ & $\mathrm{X}$ & 5,4 \\
Playa Blanca & $\mathrm{X}$ & $\mathrm{X}$ & 13,4 \\
Lobitos & $\mathrm{X}$ & $\mathrm{X}$ & 20,0 \\
Los Verdes & $\mathrm{B}$ & $\mathrm{X}$ & 21,0 \\
Pozo Toyo & $\mathrm{B}$ & $\mathrm{X}$ & 23,0 \\
Vicente Mena & $\mathrm{X}$ & $\mathrm{X}$ & 34,0 \\
Chanavayita & $\mathrm{B}$ & $\mathrm{X}$ & 54,0 \\
Punta Patache & $\mathrm{X}$ & $\mathrm{X}$ & 61,5 \\
Punta Pabellón de Pica & $\mathrm{X}$ & & 75,0 \\
\hline
\end{tabular}


habita con otros chitones, como Tonicia elegans var lineolata Frembly, 1828, Chiton cumingsi Frembly, 1827 e Ischnochiton pusio (Sowerby, 1832). Pero no se observó la presencia de Calloplax vivipara Plate, 1902, que fue recolectada anteriormente con C. pulchellus en el sector de Pozo Toyo (Ferreira, 1978). Sin embargo, la información proporcionada por Ferreira (1978) sobre C. vivipara no fue considerada en el listado de chitones chilenos realizado por Valdovinos (1999), lo cual requiere una revisión posterior.

En total se recolectaron 35 individuos en el sector de Los Verdes (12 ind), Pozo Toyo (13 ind) y Chanavayita (10 ind). En total se observaron 60, 55 y 45 individuos en cada sector respectivamente. Los ejemplares recolectados en Chanavayita presentaron una apariencia más rojiza que los ejemplares recolectados en los otros dos sitios, lo cual se debería principalmente a microalgas adheridas a sus placas. Los individuos estudiados sirvieron para realizar un análisis morfológico, que incluyó las características de las placas y del área superior del cinturón paleal.

Diagnosis: animales entre 5 y $12 \mathrm{~mm}$ de longitud, color desde blanco, blanco-amarillento a naranja pálido, con 8-14 costillas radiales en la placa cefálica. Las placas centrales son rectangulares con dos costillas en las áreas laterales que se proyectan desde el ápice y se hacen más gruesas hacia la parte inferior, débilmente granuladas (Fig. 1). Área pleural con numerosos agujeros que son más pequeños hacia el seno yugal, que es de color blanco lechoso; elevación dorsal $c a .0,3 \mathrm{~cm}$. Articulamentum color blanco, apófises cortas, triangulares y translúcidas. Mucro central, zona ante-mucronal con numerosos agujeros y zona post-mucronal cóncava con 8-11 costillas radiales débilmente granuladas. Cinturón amplio, color blanco a blanco-amarillento con numerosas escamas en la parte superior altamente imbricadas ( ${ }^{3} 0,114 \mathrm{~mm}$ longitud); las escamas tienen numerosos ribetes subgranulosos que terminan en diminutos glóbulos esféricos. Zona ventral del cinturón con escamas rectangulares altamente sobrelapadas. Borde inferior del cinturón con finas espículas translucidas ( ${ }^{3} 0,057 \mathrm{~mm}$ de longitud), estriadas, que se proyectan fuera del cinturón y se observan dorsalmente en ejemplares húmedos o vivos. Diente lateral mayor con una capa dental unicúspide aguzado en su extremo. Ctenidios o branquias holobranquial, abanal (según Kaas \& van Belle, 1994).

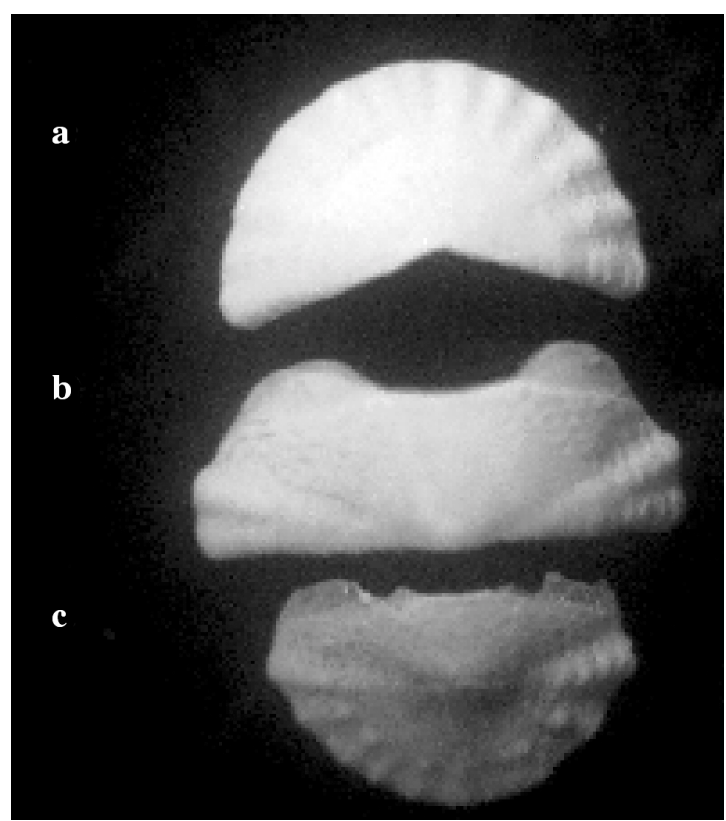

Figura 1. Callistochiton pulchellus. Vista dorsal de a) placa cefálica, b) placa central IV, c) placa VIII.

Figure 1. Callistochiton pulchellus. Dorsal view of a) cephalic plate, b) central plate IV, c) plate VIII.

Distribución: $17^{\circ}$ a $22^{\circ} \mathrm{S}$ (Islay, Perú a Pozo Toyo, Chile) e islas Galápagos (fide Kaas \& van Belle, 1994).

C. pulchellus (Gray, 1828) sería la única especie representante de este género en nuestras costas y la de distribución más austral, dado que Callistochiton viviparus Plate, 1902 (Boudet, 1945; Leloup, 1956; Stuardo, 1959) fue reubicada en el género Calloplax Thiele, 1909 por Ferreira (1978). C. vivipara difiere de $C$. pulchellus en la presencia 8-9 costillas radiales fuertes en la placa cefálica, las cuales en $C$. pulchellus son de 8-15 (generalmente 12) y débilmente marcadas; gránulos fuertemente marcados en las costillas de las áreas laterales que son ocasionales en $C$. pulchellus, y finas espinas en la parte media del cinturón que en $C$. pulchellus no se observan. Además, las placas centrales de $C$. pulchellus son más rectangulares que las de $C$. vivipara. Otras características del aparato radular de estas especies son también de interés en su diferenciación, por ejemplo la presencia de un diente central unicúspide en $C$. pulchellus, mientras que en $C$. vivipara es tricúspide (Ferreira, 1978; Kaas \& van Belle, 1994). Aunque se determinó un nuevo límite 
sur para C. pulchellus en el sector de Chanavayita, 52 km más al sur de lo señalado por Ferreira (1978), es probable que esta especie pueda ser observada en otros sectores protegidos del oleaje de la zona norte del país.

\section{AGRADECIMIENTOS}

El autor agradece las correcciones iniciales realizadas a la presente nota por el Sr. Guillermo Guzmán (Museo del Mar, UNAP), y el apoyo bibliográfico del Dr. Enrico Shwabe (Museo de Zoología de Munich). El trabajo en terreno fue beneficiado enormemente por la ayuda de la Srta. Helga Vergara. Finalmente, el autor agradece el patrocinio y permanente estimulación en el ámbito de la ciencia de la ecología, al Dr. Sergio Navarrete (ECIM-PUC). Se agradece el apoyo de MECESUP durante el desarrollo del manuscrito.

\section{REFERENCIAS}

Boudet, I. 1945. Los quitones chilenos. Rev. Chil. Hist. Nat., 48: 122-140.

Ferreira, A. 1978. The genus Calloplax Thiele, 1909 (Mollusca: Polyplacophora) in the Americas. Bull. South. Calif. Acad. Sci., 77: 56-64.
Ferreira, A. 1979. The genus Callistochiton Dall, 1879 (Mollusca: Polyplacophora) in the Eastern Pacific, with the description of a new species. Veliger, 21: 444-466.

Kaas, P. \& R. van Belle. 1994. Monograph of living chitons. Vol 5. Suborder Ischnochitonina: Ischnochitonidae: Ischnochitoninae (concluded), Callistoplacinae; Mopaliidae. E.J. Brill. Leiden, pp. 161-163.

Keen, M. 1971. Sea shells of tropical West America: marine molluscs from lower California to Colombia. Stanford University Press, Stanford, pp. 514530 .

Leloup, E. 1956. Polyplacophora. The Lund University Chile Expedition Reports 1948-1949, 95 pp.

Marincovich, L. 1973. Intertidal mollusks of Iquique, Chile. Natural History Museum. Los Angeles Coun. Sci. Bull., 16: 42-49.

Stuardo, J. 1959. Ensayo de una clave para familias y géneros chilenos de Polyplacophora, con generalidades del grupo e inclusión de algunas especies comunes. Invest. Zool. Chil., 5: 139-148.

Valdovinos, C. 1999. Biodiversidad de moluscos chilenos: base de datos taxonómica y distribucional. Gayana, 63: 111-164. 